Proceedings of the International Congress on Advances in Applied Physics and Materials Science, Antalya 2011

\title{
Stability Analysis of FGM Layered Shells in the Surrounding Medium
}

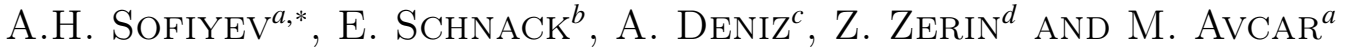 \\ ${ }^{a}$ Department of Civil Engineering, Suleyman Demirel University, Isparta, Turkey \\ ${ }^{b}$ Institute of Solid Mechanics of Karlsruhe Technology Institute, Karlsruhe, Germany \\ ${ }^{c}$ Department of Mathematics, Usak University, Usak, Turkey \\ ${ }^{d}$ Department of Civil Engineering, Ondokuz Mayıs University, Samsun, Turkey
}

\begin{abstract}
In this study, the stability analysis of three-layered shells containing a functionally graded material layer in the surrounding medium and subjected to the uniform lateral pressure is investigated. The surrounding elastic medium is modeled as a Pasternak foundation. The dimensionless critical lateral pressures of three-layered functionally graded material shells with and without elastic foundations are obtained. Effects of compositional profiles and elastic foundation on the dimensionless critical lateral pressures have been studied.
\end{abstract}

PACS: 61.43.Bn, 62.20.mq

\section{Introduction}

In recent years, functionally graded materials (FGMs) have been developed due to their excellent mechanical and thermal properties. FGMs can be structured from two or more materials to meet specific purposes of engineering. These materials have emerged from the need to enhance material performance [1]. The static and dynamic interaction of FGM shells with the elastic medium is a problem of current importance because circular shells are widely used in various engineering applications such as gas pipelines and tubes, rockets and missiles, and other civil and mechanical engineering structures. Stability and vibration analyses of FGMs are quite limited, especially of those on elastic foundations [2,3]. Investigations of stability of three-layered shells containing an FGM layer under various loads without elastic foundations have also been reported [4-6]. To the best of authors' knowledge, no work has been done on the bucking behavior of three-layered shells containing an FGM layer under the uniform lateral pressure and resting on the Pasternak type elastic foundation. When layered conical shells made of FGMs are considered, the problem gets even more complicated. Therefore, it is very important to develop an accurate, reliable analysis towards the understanding of the stability characteristics of layered FGM shells. In the present work, an attempt is made to address this problem.

\section{Basic equations}

Consider a generic three-layered truncated conical shell made up of an FGM layer in between the fully ceramic and fully metal layers subjected to the uniform lateral

\footnotetext{
* corresponding author; e-mail: asofiyev@mmf.sdu.edu.tr
}

pressure $P$, as shown in Fig. 1. In Fig. 1, (1) is fully ceramic layer, (2) is FGM layer and (3) is fully metal layer. The ceramic and metal layers are assumed to be homogeneous and isotropic. Here $h=h_{1}+h_{2}$ is the total thickness of the shell, $2 a$ is the thickness of the FGM layer, $R_{1}$ and $R_{2}$ indicate the radii of the cone at its lower and upper ends, respectively. $L$ is the length and $\gamma$ is the semi-vertex angle of the conical shell. $S_{1}$ and $S_{2}$ are the distances from the vertex to the lower and upper bases, respectively. A set of curvilinear coordinates are presented in Fig. 1.

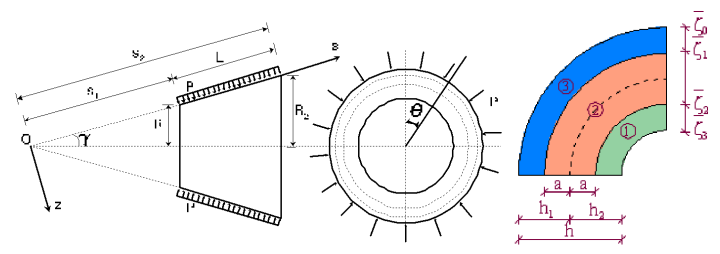

Fig. 1. Geometry of the three-layered conical shell containing an FGM layer.

The compositional gradation of the FG layer is defined by the volume fraction of the ceramic phase. Here, the following functions of $z$ will be considered for $V_{\mathrm{c}}[1,2]$ :

$$
\begin{aligned}
& V_{\mathrm{c}}=\bar{z}+0.5, \quad V_{\mathrm{c}}=(\bar{z}+0.5)^{2}, \\
& V_{\mathrm{c}}=1-(0.5-\bar{z})^{2}, \quad \bar{z}=z / 2 a .
\end{aligned}
$$

For such cases, variations of the Young modulus and Poisson's ratio in the three-layered system are given as [4-6]:

$$
\begin{aligned}
& E(\bar{z}), v(\bar{z})= \\
& \left\{\begin{array}{r}
E_{0 \mathrm{~m}}, v_{0 \mathrm{~m}} \text { for }-h_{1} \leq z \leq-a ; E_{0 \mathrm{c}}, v_{0 \mathrm{c}} \\
\text { for } a \leq z \leq h_{2}, \\
E_{\mathrm{fg}}=E_{\mathrm{m}}+\left(E_{\mathrm{c}}-E_{\mathrm{m}}\right) V_{\mathrm{c}}, v_{\mathrm{fg}}=v_{\mathrm{m}}+\left(v_{\mathrm{c}}-v_{\mathrm{m}}\right) V_{\mathrm{c}} \\
\text { for }-a \leq z \leq a,
\end{array}\right.
\end{aligned}
$$


where $E_{0 \mathrm{~m}}, v_{0 \mathrm{~m}}$ and $E_{0 \mathrm{c}}, v_{0 \mathrm{c}}$ are the Young modulus and Poisson's ratio of the metal and ceramic materials, respectively, $E_{\mathrm{m}}, v_{\mathrm{m}}$ and $E_{\mathrm{c}}, v_{\mathrm{c}}$ are the Young modulus and Poisson's ratio of the metal and ceramic surfaces of the FGM conical shell, respectively.

The layered FGM truncated conical shell is resting on an elastic foundation. For the elastic foundation, it is assumed the two-parameter elastic foundation. The foundation medium is assumed to be linear, homogeneous and isotropic. The bonding between the truncated conical shell and the foundation is perfect and frictionless $[2,3]$.

The modified Donnell type stability and compatibility equations of three-layered truncated conical shell containing an FGM layer subjected to the lateral pressure and resting on the Pasternak type elastic foundation, can be obtained as [3]:

$$
\begin{aligned}
& C_{2} \mathrm{e}^{2 x}\left(\frac{\partial^{4} \Phi_{1}}{\partial x^{4}}-4 \frac{\partial^{3} \Phi_{1}}{\partial x^{3}}+4 \frac{\partial^{2} \Phi_{1}}{\partial x^{2}}+\frac{\partial^{4} \Phi_{1}}{\partial \varphi^{4}}+2 \frac{\partial^{2} \Phi_{1}}{\partial \varphi^{2}}\right) \\
& +2\left(C_{1}-C_{5}\right) \mathrm{e}^{2 x}\left(\frac{\partial^{4} \Phi_{1}}{\partial x^{2} \partial \varphi^{2}}-2 \frac{\partial^{3} \Phi_{1}}{\partial x \partial \varphi^{2}}+\frac{\partial^{2} \Phi_{1}}{\partial \varphi^{2}}\right) \\
& +\left(\frac{\partial^{2} \Phi_{1}}{\partial x^{2}}+3 \frac{\partial \Phi_{1}}{\partial x}+2 \Phi_{1}\right) \mathrm{e}^{3 x} S_{1} \cot \gamma \\
& -S_{1}^{3} \mathrm{e}^{3 x} P \tan \gamma\left(\frac{\partial^{2} w}{\partial \varphi^{2}}+\frac{\partial w}{\partial x}\right)-S_{1}^{4} \mathrm{e}^{4 x} K_{w} w \\
& +K_{p} S_{1}^{2} \mathrm{e}^{2 x}\left(\frac{\partial^{2} w}{\partial x^{2}}+\frac{\partial^{2} w}{\partial \varphi^{2}}\right) \\
& +C_{3}\left(\frac{\partial^{4} w}{\partial \varphi^{4}}+\frac{\partial^{4} w}{\partial x^{4}}-4 \frac{\partial^{3} w}{\partial x^{3}}+4 \frac{\partial^{2} w}{\partial x^{2}}+2 \frac{\partial^{2} w}{\partial \varphi^{2}}\right) \\
& -2\left(B_{6}-B_{3}\right)\left(\frac{\partial^{4} w}{\partial x^{2} \partial \varphi^{2}}-2 \frac{\partial^{2} w}{\partial x^{2}}-\frac{\partial w}{\partial x}\right)=0 \\
& +2\left(C_{4}+C_{6}\right)\left(\frac{\partial^{4} w}{\partial x^{2} \partial \varphi^{2}}-2 \frac{\partial^{4} w}{\partial x \partial \varphi^{2}}+\frac{\partial^{2} w}{\partial \varphi^{2}}\right)=0 \\
& +2\left(B_{5}+B_{2}\right) \mathrm{e}^{2 x}\left(\frac{\partial^{4} \Phi_{1}}{\partial x^{2} \partial \varphi^{2}}+2 \frac{\partial^{3} w}{\partial x \partial \varphi^{2}}+\frac{\partial^{2} \Phi_{1}}{\partial \varphi^{2}}\right) \\
& B_{1} \mathrm{e}^{2 x}\left(\frac{\partial^{4} \Phi_{1}}{\partial x^{4}}+4 \frac{\partial^{3} \Phi_{1}}{\partial x^{3}}+4 \frac{\partial^{2} \Phi_{1}}{\partial x^{2}}+\frac{\partial^{4} \Phi_{1}}{\partial \varphi^{4}}+2 \frac{\partial^{2} w}{\partial \varphi^{2}}\right)
\end{aligned}
$$

where $\varphi=\theta \sin \gamma, \Phi_{1}=\Phi \mathrm{e}^{-2 x}, S_{1}=S \mathrm{e}^{-x}, K_{w}\left[\mathrm{~N} / \mathrm{m}^{3}\right]$ is the modulus of subgrade reaction for the foundation, $K_{p}[\mathrm{~N} / \mathrm{m}]$ is the shear modulus of the subgrade $[2,3]$,
$C_{j}, B_{j}(j=1,2 \ldots, 6)$ are depending on the materials properties and characteristics of the three-layered shell containing an FGM layer, see Refs. [4-6].

\section{Solution of the basic equations}

Assuming that the FGM truncated conical shell is simply supported at both ends. The solution of Eq. (4) is sought in the following form:

$w=A \mathrm{e}^{x} \sin \beta_{1} x \sin \beta_{2} \varphi$,

where $A$ is amplitude and $\beta_{1}=m \pi / x_{0}, \beta_{2}=n / \sin \gamma$, $x_{0}=\ln \left(S_{2} / S_{1}\right)$.

Substituting expression (5) into Eq. (4), the particular solution is obtained as follows:

$$
\begin{gathered}
\Phi_{1}=A\left(K_{1} \sin \beta_{1} x+K_{2} \cos \beta_{1} x\right. \\
\left.+K_{3} \mathrm{e}^{-x} \sin \beta_{1} x\right) \sin \beta_{2} \varphi,
\end{gathered}
$$

where $K_{i}(i=1,2,3)$ are depending on the material properties and shell characteristics, see Ref. [5].

Substituting Eqs. (5) and (6) into Eq. (4) and applying the Galerkin method, in the ranges $0 \leq \varphi \leq 2 \pi \sin \gamma$ and $0 \leq x \leq x_{0}$, after integrating for the dimensionless critical lateral pressure of the three-layered conical shell containing an FGM layer on the Pasternak type elastic foundation, the following equation is obtained:

$$
P_{L 1 w p}^{\mathrm{cr}}=\frac{U_{1}+K_{w} U_{2}+K_{p} U_{3}}{U_{4} E_{0 \mathrm{c}}},
$$

where $U_{i}(i=1,2,3,4)$ are depending on the materials and shell characteristics [3].

When $K_{p}=0$, from Eq. (7), the expression for $P_{L 1 w}^{\mathrm{cr}}$ of the three-layered FGM conical shell on the Winkler foundation are obtained. When $K_{p}=K_{w}=0$, from Eq. (7), the expression for $P_{L 1}^{\mathrm{cr}}$ of the three-layered FGM conical shell without an elastic foundation are obtained. When $\gamma \rightarrow 0^{\circ}$, from Eq. (7), the expressions for critical lateral pressure of the three-layered FGM cylindrical shells with and without a Pasternak type elastic foundation are obtained.

\section{Numerical computations and results}

Variations of the dimensionless critical lateral pressures for fully metal, fully ceramic, fully FGM and $\mathrm{Ni}-$ FGM- $\mathrm{Si}_{3} \mathrm{~N}_{4}$ truncated conical and cylindrical shells for different compositional profiles with or without the Winkler or Pasternak foundations, versus the semi-vertex angle $\gamma$, the ratio of the thickness of the shell to the thickness of an FGM layer $h / 2 a$, and the ratio of the length to the small radius $L / R_{1}$, are presented in Table. As the semi-vertex angle $\gamma$ and the ratio $L / R_{1}$ increases, the values of $P_{L 1}^{\mathrm{cr}}, P_{L 1 w}^{\mathrm{cr}}, P_{L 1 w p}^{\mathrm{cr}}$ decrease, whereas as the ratio $h /(2 a)$ increases, $P_{L 1}^{\mathrm{cr}}, P_{L 1 w}^{\mathrm{cr}}, P_{L 1 w p}^{\mathrm{cr}}$ increase for three-layered shells containing an FGM layer with or without elastic foundations. The effect of compositional profiles on the values of $P_{L 1}^{\mathrm{cr}}$ is remains constant, whereas, the effect of the compositional profiles on the values of 
$P_{L 1 w}^{\mathrm{cr}}, P_{L 1 w p}^{\mathrm{cr}}$ decrease, as the semi-vertex angle $\gamma$ and $L / R_{1}$ increase.

The effect of compositional profiles on is the same, whereas this effect on the values ofdecrease, as increase. The effect of elastic foundations on the dimensionless critical lateral pressure increases, as the values of $\gamma$ and $L / R_{1}$ increase. As the ratio $h /(2 a)=1$, the three-layered shells containing an FGM layer are transformed to fully FGM shells. Furthermore, the values of $P_{L 1}^{\mathrm{cr}}, P_{L 1 w}^{\mathrm{cr}}, P_{L 1 w p}^{\mathrm{cr}}$ corresponding to $\gamma \rightarrow 0^{\circ}$ related to the cylindrical shell. The values of dimensionless critical lateral pressures of the truncated conical shell with and without elastic foundations are lower than values of the cylindrical shell. It is clear that the effect of the Pasternak type elastic foundation on the dimensionless critical lateral pressure is higher than the Winkler elastic foundation.

TABLE

Variations of $P_{L 1}^{\mathrm{cr}}, P_{L 1 w}^{\mathrm{cr}}, P_{L 1 w p}^{\mathrm{cr}}$ for the fully ceramic, fully metal and $\mathrm{Si}_{3} \mathrm{~N}_{4}-\mathrm{FGM}-\mathrm{Ni}$ shells in the surrounding medium.

\begin{tabular}{|c|c|c|c|c|c|c|}
\hline $\begin{array}{c}K_{w}=5 \times 10^{6} \mathrm{~N} / \mathrm{m}^{3} ; \\
K_{p}=10^{5} \mathrm{~N} / \mathrm{m}\end{array}$ & \multicolumn{2}{|c|}{$P_{L 1}^{\mathrm{cr}}\left(n_{\mathrm{cr}}\right)$} & \multicolumn{2}{|c|}{$P_{L 1 w}^{\mathrm{cr}}\left(n_{\mathrm{cr}}\right)$} & \multicolumn{2}{|c|}{$P_{L 1 w p}^{\mathrm{cr}}\left(n_{\mathrm{cr}}\right)$} \\
\hline $\begin{array}{c}h /(2 a) \\
\left(R_{1} / h=100\right. \\
L / R_{1}=2 \\
\left.\gamma=30^{\circ}\right)\end{array}$ & $\mathrm{Si}_{3} \mathrm{~N}_{4}$ & $\begin{array}{c}\mathrm{Ni}-\mathrm{FGM}-\mathrm{Si}_{3} \mathrm{~N}_{4} \\
\text { quad. }\end{array}$ & $\mathrm{Si}_{3} \mathrm{~N}_{4}$ & $\begin{array}{c}\mathrm{Ni}-\mathrm{FGM}-\mathrm{Si}_{3} \mathrm{~N}_{4} \\
\text { quad. }\end{array}$ & $\mathrm{Si}_{3} \mathrm{~N}_{4}$ & $\begin{array}{c}\mathrm{Ni}-\mathrm{FGM}-\mathrm{Si}_{3} \mathrm{~N}_{4} \\
\text { quad. }\end{array}$ \\
\hline 1 & & $0.155(8)$ & & $0.185(8)$ & & $0.203(8)$ \\
\hline 1.5 & $0.211(8)$ & $0.168(8)$ & $0.241(1,8)$ & $0.198(8)$ & $0.258(8)$ & $0.215(8)$ \\
\hline 5 & & $0.175(8)$ & & $0.204(8)$ & & $0.222(8)$ \\
\hline $\begin{array}{c}L / R_{1} \\
(h / 2 a=2, \\
R_{1} / h=100, \\
\left.\gamma=30^{\circ}\right)\end{array}$ & $\mathrm{Ni}$ & $\begin{array}{l}\mathrm{Ni}-\mathrm{FGM}-\mathrm{Si}_{3} \mathrm{~N}_{4} \\
\text { inv. quad. }\end{array}$ & $\mathrm{Ni}$ & $\begin{array}{l}\mathrm{Ni}-\mathrm{FGM}-\mathrm{Si}_{3} \mathrm{~N}_{4} \\
\text { inv. quad. }\end{array}$ & $\mathrm{Ni}$ & $\begin{array}{c}\mathrm{Ni}-\mathrm{FGM}-\mathrm{Si}_{3} \mathrm{~N}_{4} \\
\text { inv. quad. }\end{array}$ \\
\hline 1 & $0.401(9)$ & $0.504(9)$ & $0.420(1,9)$ & $0.519(10)$ & $0.444(10)$ & $0.542(10)$ \\
\hline 2 & $0.140(8)$ & $0.175(8)$ & $0.170(1,8)$ & $0.205(8)$ & $0.188(8)$ & $0.223(8)$ \\
\hline 3 & $0.071(7)$ & $0.090(7)$ & $0.112(1,8)$ & $0.130(8)$ & $0.126(8)$ & $0.145(8)$ \\
\hline $\begin{array}{c}\gamma \\
\left(R_{1} / h=100\right. \\
L / R_{1}=2 \\
h / 2 a=2)\end{array}$ & $\mathrm{Si}_{3} \mathrm{~N}_{4}$ & $\begin{array}{c}\mathrm{Ni}-\mathrm{FGM}-\mathrm{Si}_{3} \mathrm{~N}_{4} \\
\operatorname{lin}\end{array}$ & $\mathrm{Si}_{3} \mathrm{~N}_{4}$ & $\begin{array}{c}\mathrm{Ni}-\mathrm{FGM}-\mathrm{Si}_{3} \mathrm{~N}_{4} \\
\operatorname{lin}\end{array}$ & $\mathrm{Si}_{3} \mathrm{~N}_{4}$ & $\begin{array}{c}\mathrm{Ni}-\mathrm{FGM}-\mathrm{Si}_{3} \mathrm{~N}_{4} \\
\text { lin }\end{array}$ \\
\hline $0^{\circ}(\mathrm{Cyl})$ & $0.478(6)$ & $0.393(6)$ & $0.518(1,6)$ & $0.432(6)$ & $0.548(6)$ & $0.463(6)$ \\
\hline $30^{\circ}$ & $0.210(8)$ & $0.173(8)$ & $0.241(1,8)$ & $0.203(8)$ & $0.258(8)$ & $0.221(8)$ \\
\hline $45^{\circ}$ & $0.127(8)$ & $0.105(8)$ & $0.1551(1,8)$ & $0.132(9)$ & $0.168(8)$ & $0.145(9)$ \\
\hline $60^{\circ}$ & $0.067(8)$ & $0.055(8)$ & $0.088(1,8)$ & $0.076(8)$ & $0.097(8)$ & $0.085(9)$ \\
\hline
\end{tabular}

\section{Conclusions}

In this study, the stability analysis of three-layered shells containing an FGM layer in the surrounding medium and subjected to the uniform lateral pressure is investigated. The surrounding elastic medium is modeled as a Pasternak foundation. The dimensionless critical lateral pressures of three-layered FGM shells with and without elastic foundations are obtained. Effects of compositional profiles and elastic foundation on the critical lateral pressures have been studied.

\section{Acknowledgments}

This study is supported by the TUBITAK under project number 110M695. The first, third and fifth au- thors thank to TUBITAK for the support of the project number 110M695.

\section{References}

[1] M. Koizumi, Ceramic Trans., Funct. Gradient Mater. 34, 3 (1993).

[2] H.S. Shen, Int. Mech. Sci. 51, 372 (2009).

[3] A.H. Sofiyev, Int. J. Pres. Ves. Pip. 87, 753 (2010).

[4] S.R. Li, R.C. Batra, Thin-Walled Struct. 44, 1039 (2006).

[5] A.H. Sofiyev, Z. Zerin, A. Korkmaz, Compos. Struct. 85, 105 (2008).

[6] S.R. Li, X.H. Fub, R.C. Batra, Mech. Res. Commun. 37, 577 (2010). 